\title{
Application of Advanced Spectral Analysis for Rehabilitation Progress Estimation Concerning Patients after Ischemic Brain Stroke
}

\author{
Ewaryst Tkacz, Ivo Provaznik, and Paweł Kostka
}

\begin{abstract}
It is possible to observe recently that application of the simple, known tools for biomedical signals processing is no longer justified. The problems under analysis frequently require application of more and more sophisticated tools. This is the case in attempt of estimation of rehabilitation progress referring to patients after ischemic brain stroke. Classic approach apply acquisition of EEG signal and following that application of different signal processing tools for analysis and classification of patients after ischemic brain stroke using e.g. Scandinavian scale. In this paper the totally different, indirect approach is discussed. Instead of EEG the application of relatively easier for acquisition signal such as HRV is applied. However, again classic analysis methods well established in Task Force report such as power spectral estimation present several disadvantages like e.g. loss of phase information. The trends turn the attention into more sophisticated methods and one among them concerns application of the second order statistics and following that calculation of both bispectrum and bicoherence. The presented considerations are in fact first serious study after years of existing of mentioned tool. However the difficulties in calculations put that tool into the background of signal processing method. The general trend in current analysis of such signal is focused on application of sort of hybrid method taking into account methods from different areas and creating in that way a novel approach by attempt of application of indirect method allowing effective conclusions.
\end{abstract}

Index Terms-ECG, EEG, HRV, higher order spectral analysis.

\section{INTRODUCTION}

Biomedical signals are in most cases highly complex and fortunately understandably nonlinear. This concerns for example firing of neurons, beating of the heart or breathing. These signals arise out of a multitude of interconnected elements comprising of the human body. Mentioned elements are bounded and the connections are rather weakly coupled across all elements. Signal propagation processes vary in time scales ranging from nanoseconds for molecular motion to gross observable behavior in term of days and years. This is in fact the reason why biomedical systems are

Manuscript received September 10, 2013; revised November 13, 2013.

E. Tkacz is with the Department of Biosensors and Biomedical Signals Processing, Faculty of Biomedical Engineering, Silesian University of Technology, Gliwice, Poland. He is also with Institute of Biomedical Engineering, Brno University of Technology, Brno, Czech Republic (e-mail: etkacz@polsl.pl ).

I. Provaznik is with the Institute of Biomedical Engineering, Brno University of Technology, Brno, Czech Republic (e-mail: provaznik@feec.vutbr.cz ).

P. Kostka is with the Department of Biosensors and Biomedical Signals Processing, Faculty of Biomedical Engineering, Silesian University of Technology, Gliwice, Poland (e-mail:pawel.kostka@ polsl.pl). highly nonstationary [1], [2].

The fundamental nature of the brain's electrical activity remains unknown. Therefore a linear stochastic model of the brain as well as pure spectral analysis of its activity seems to appear nonsufficient as signals recorded are usually associated with some noise and artifacts, which in most cases highly contaminate obtained results of measurements and further analysis [3]. In this paper we have decided to present an indirect approach, less stress-causing for the individual patient and on the other hand much common and simpler incomparison to the EEG recordings. This concerns application of HRV recording and analysis. Having created a suitable database with ECG recording performed with the help of Holter method registration we started to apply a bit more sophisticated analysis such as calculation of bispectra and bicoherence [4]-[8].

\section{MATERIAL AND METHODS}

\section{A. Selecting of Patients}

There have been 21 patients under examinations for whom the ischemic brain stroke (IBS) has been reported. Each patient had mentioned above Holter method ECG signal registration during or around first day after IBS, tenth day and finally sixty days. For 5 patients the last registration has not been performed due to their death. Finally the databaseconsisting of 16 patients has been created for further analysis and processing.

\section{B. Preprocessing to the Main Calculations}

The significant reason for preprocessing of registered signals before the main calculations comes out from the rather poor quality of registered signals especially during first day after IBS and tenth day. It can be partially explained by the unpredictable behavior of patients as well as appearance of additional symptoms associated with the IBS itself. The preprocessing procedure incorporated visual inspection of all the registrations, modeling of the ECG signal to create a suitable representation of the HRV with the help of both frequency deconvolution method and application of cubic splines interpolation and resampling with the frequency of 4 $\mathrm{Hz}$ according to the HRV Task Force recommendations.

\section{MATH}

The important limitation of a power spectrum in case of signal analysis comes from a fact that is doesn't carry information about phase. However, such information can be obtained from higher-order polyspectra. 
All Gaussian random effects can be cancelled by use of the higher-order moments and an amount of a remaining nonlinear coupling can be measured by a bispectrum or its normalized form-bicoherence. Below, there are basic necessary mathematical considerations. Fourier transform of the recorded signal $f(t)$ is given by:

$$
f^{\prime}(w)=\int_{-\infty}^{\infty} f(t) e^{-i \omega t} d t
$$

Next, its power spectrum can be easy calculated and therefore is given by:

$$
\operatorname{Pf}(w)=\left|f^{\prime}(w)\right|^{2} .
$$

A natural estimate of the bispectrum $\left(B_{x}\right)$ is then given by a Fourier transform of the third-order cumulant sequence $\left(C_{x}\right)$ :

$$
B_{x}\left(f_{1}, f_{2}\right)=\sum_{k=-\infty}^{\infty} \sum_{l=-\infty}^{\infty} C_{x}(k, l) e^{-i 2 \pi f_{1} k} e^{-i 2 \pi f_{2} l} .
$$

This leads to another important term - bicoherence. It is a normalized bispectrum and can be described as anount of phase-coupling in a signal or between two signals. Finally, phase-coupling is defined as an estimate proportion of energy in every possible pair of frequency components that satisfies the definition of quadratic phase-coupling. It means that phase of component at $\mathrm{f}_{3}$ (assuming that $f_{3}=f_{1}+f_{2}$ ) is equal to phase of $f_{1}+$ phase of $\mathrm{f}_{2}$ as the whole operation is additive and therefore a superposition theorem can be applied [5].

Knowing these basic explanations, let us introduce the idea of this paper. We present the results of bispectral analysis of three HRV recordings concerning all the patients in created database respectively at first day after IBS, tenth day after IBS and finally sixty days after IBS.

In each case the record from a 24-hour Holter's monitoring was limited to 50000 samples in attempt to facilitate visual assessment of the results. In order to avoid the adverse effects of the external noise the efficient filtering procedure has been applied. The bispectra have been computed using a direct (FFT-based) estimation method implemented in Matlab's Higher-Order Spectral Analysis Toolbox. The overlap factor has been set to 0 and number of samples per segment respectively to 100 . For each of the analyzed signals we present bispectral plot along with a diagonal slice of an obtained bispectrum. It is worth to mention, at this stage, that the time of calculation has not been critical and using an oldMatlab tools, unfortunately not available in the newest Matlab version, we were able to obtain the final results using standard computing facilities (i.e. i7 processor with $3.03 \mathrm{GHz}$ clock) in less than 4 minutes.

\section{DESCRIPTION OF INVESTIGATION METHODOLOGY}

Fig. 1, Fig. 3 and Fig. 5 present the calculated bispectra for the third patient from the created and described database, where registration procedure has been preserved. It can be interpreted that critical moment in the rehabilitation procedure appeared somewhere around tenth day after IBS due to most irregular shape of the presented bispectrum. Fig. 2, Fig. 4, and Fig. 6 present a section or diagonal slice of HRV's along respective bispectrum. However, a detailed numerical results of the surface under the bispectrum curve both Table I and Table II, where Table I presents results concerning of investigated patients and Table II summarizes results in respective periods of examinations.

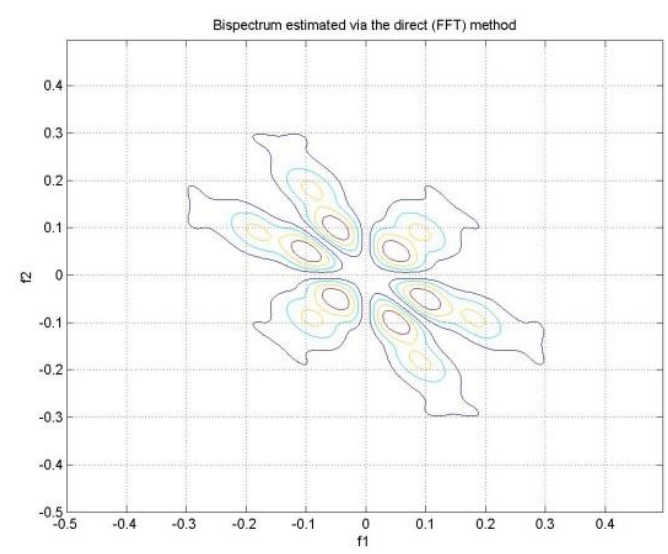

Fig. 1. Patient 3: HRV's bispectrum (record 1).

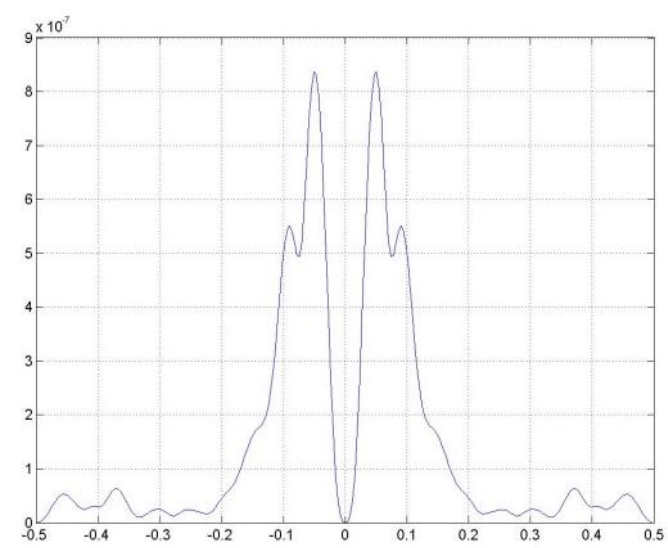

Fig. 2. Patient 3: diagonal slice of HRV's bispectrum (record 1).

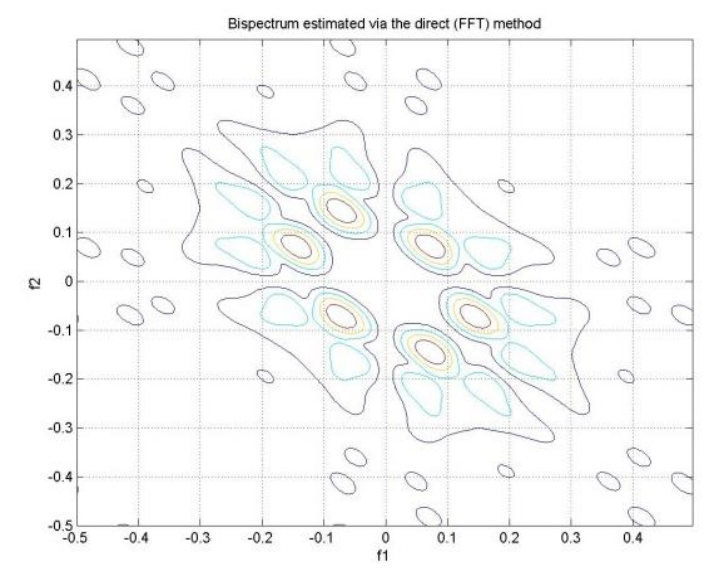

Fig. 3. Patient 3: HRV's bispectrum (record 2)

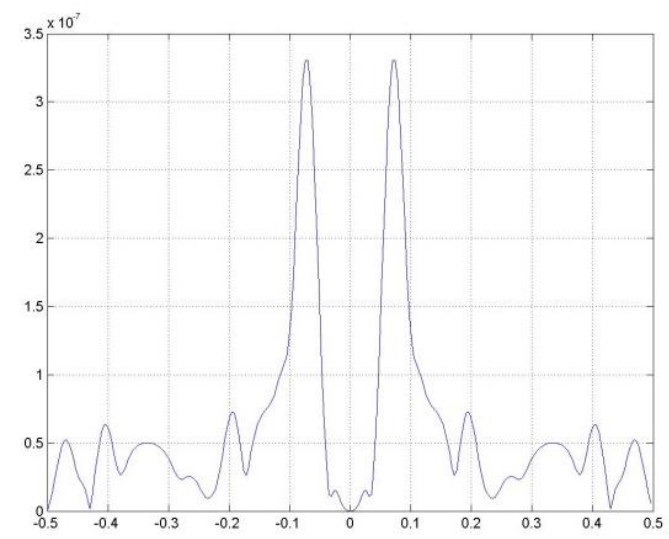

Fig. 4. Patient 3: diagonal slice of HRV's bispectrum (record 2). 


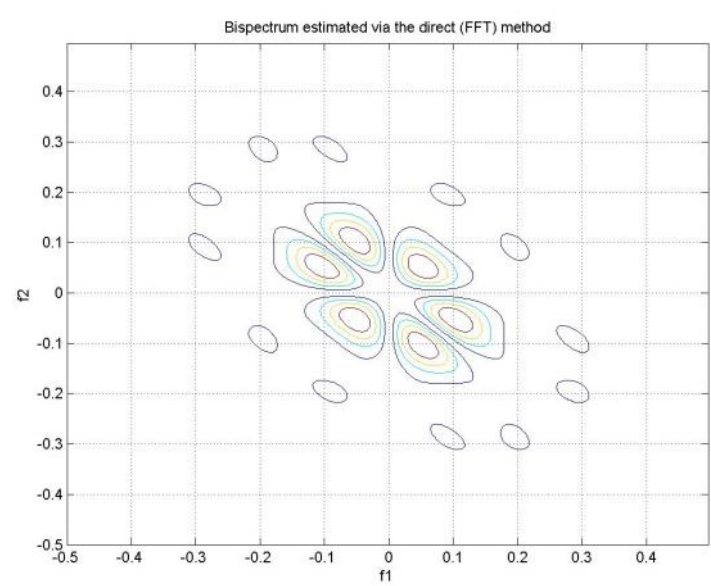

Fig. 5. Patient 3: HRV's bispectrum (record 3).

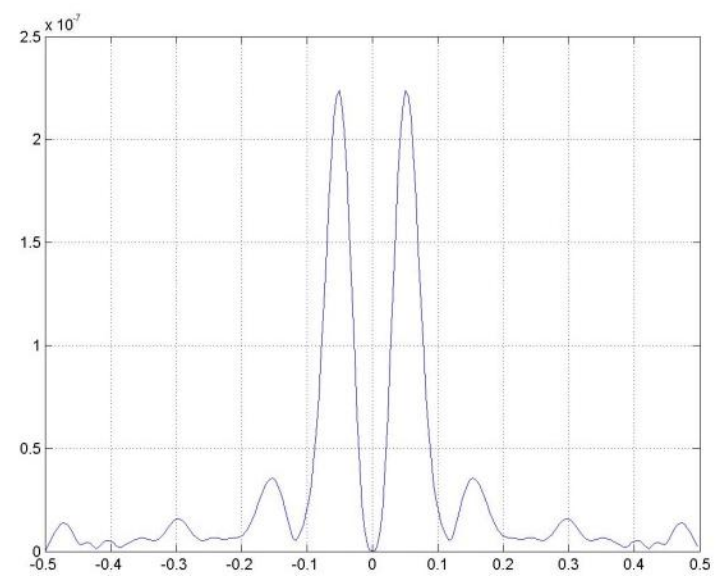

Fig. 6. Patient 3: diagonal slice of HRV's bispectrum (record 3).

TABLE I: THE SURFACE UNDER THE CURVE OF BISPECTRUM’S DIAGONAL

\begin{tabular}{|c|c|c|c|}
\hline \multirow{2}{*}{ Patient } & Record 1 & Record 2 & Record 3 \\
\hline 1 & $3,03 \mathrm{E}-08$ & $1,23 \mathrm{E}-07$ & $5,07 \mathrm{E}-08$ \\
\hline 3 & $1,49 \mathrm{E}-07$ & $6,23 \mathrm{E}-08$ & $2,95 \mathrm{E}-08$ \\
\hline 4 & $1,17 \mathrm{E}-09$ & $5,75 \mathrm{E}-10$ & $2,95 \mathrm{E}-10$ \\
\hline 5 & $1,88 \mathrm{E}-09$ & $2,28 \mathrm{E}-08$ & $3,64 \mathrm{E}-09$ \\
\hline 6 & $6,09 \mathrm{E}-09$ & $3,02 \mathrm{E}-08$ & $6,71 \mathrm{E}-09$ \\
\hline 7 & $1,21 \mathrm{E}-07$ & $3,54 \mathrm{E}-08$ & $5,74 \mathrm{E}-08$ \\
\hline 8 & $3,28 \mathrm{E}-08$ & $2,23 \mathrm{E}-07$ & $4,17 \mathrm{E}-08$ \\
\hline 9 & $1,92 \mathrm{E}-07$ & $4,28 \mathrm{E}-08$ & $3,85 \mathrm{E}-08$ \\
\hline 10 & $1,64 \mathrm{E}-09$ & $4,12 \mathrm{E}-10$ & $2,35 \mathrm{E}-10$ \\
\hline 11 & $1,34 \mathrm{E}-09$ & $1,28 \mathrm{E}-08$ & $2,64 \mathrm{E}-09$ \\
\hline 12 & $4,19 \mathrm{E}-09$ & $2,02 \mathrm{E}-08$ & $5,71 \mathrm{E}-09$ \\
\hline 13 & $2,11 \mathrm{E}-07$ & $3,23 \mathrm{E}-08$ & $2,85 \mathrm{E}-08$ \\
\hline 14 & $1,64 \mathrm{E}-09$ & $3,15 \mathrm{E}-10$ & $6,35 \mathrm{E}-10$ \\
\hline 15 & $3,49 \mathrm{E}-09$ & $5,28 \mathrm{E}-08$ & $2,64 \mathrm{E}-09$ \\
\hline 16 & $2,61 \mathrm{E}-07$ & $4,02 \mathrm{E}-08$ & $2,71 \mathrm{E}-09$ \\
\hline
\end{tabular}

Calculating simple power spectrum density (PSD) of the HRV signals do not produce such a result as PSD loses phase information probably important from the interpretation point of view. Therefore we suggested a novel and different way of rehabilitation progress estimation which refers to calculation of the surface under the bispectrum diagonal slice.
TABLE II: THE SURFACE UNDER THE CURVE OF BISPECTRUM'S DIAGONAL

\begin{tabular}{|c|c|c|c|}
\hline \multicolumn{5}{|c}{ SLICE-ADDITIONAL CALCULATIONS } \\
\hline Parameter & Record 1 & Record 2 & Record 3 \\
\hline Mean & $6,79 \mathrm{E}-08$ & $4,66 \mathrm{E}-08$ & $1,81 \mathrm{E}-08$ \\
\hline $\begin{array}{c}\text { Population } \\
\text { standard } \\
\text { deviation }\end{array}$ & $6,09 \mathrm{E}-09$ & $3,23 \mathrm{E}-08$ & $5,71 \mathrm{E}-09$ \\
\hline $\begin{array}{c}\text { Sample } \\
\text { standard } \\
\text { deviation }\end{array}$ & $9,59456 \mathrm{E}-08$ & $6,28289 \mathrm{E}-08$ & $2,53729 \mathrm{E}-08$ \\
\hline
\end{tabular}

\section{CONCLUSION}

As a conclusion the authors would like to stress that suggested method present preliminary results and definitely needs further investigation. However, taking into account information that most of biomedical systems are highly nonlinear the paper may be considered as a small contribution to convince researchers to return to relatively old and known methods with the new light depending upon the application field.

\section{ACKNOWLEDGMENT}

This research paper has been partially sponsored by the program project UMO-2012/07/B/ST6/01238 granted by the National Center of Science in Kraków, Poland. Therefore Authors of this work would like to express their great appreciation.

\section{REFERENCES}

[1] T. H. Bullock, J. Z. Achimowicz et al., "Bicoherence of intracranial EEG in sleep, wakefulness and seizures," Electroencephalography and Clinical Neurophysiology, vol. 103, no. 6, pp. 661-678, 1997.

[2] J. P. Pijn, J. V. Neerven, A. Noest, and F. H. L. D. Silva, "Chaos or noise in EEG signals; dependence on state and brain site," Electroencephalography and Clinical Neurophysiology, vol. 79,no. 5, pp. 371-381, 1991.

[3] J. Theiler, S. Eubank, A. Longtin, B. Galdrikian, and J. DoyneFarmer, "Testing for nonlinearity in time series: the method of surrogate data," Physica D, vol. 58, no. 1-4, pp. 77-94, 1992.

[4] J. M. Mendel, "Tutorial on higher-order statistics (spectra) in signalprocessing and system theory: Theoretical results and some applications," in Proc. IEEE, 1991, vol. 79, pp. 278-305.

[5] C. Pradhan, S. K. Jena, S. R. Nadar, and N. Pradhan, "Higher-order spectrum in understanding nonlinearity in EEG rhythms," Computational and Mathematical Methods in Medicine, vol. 2012, pp. 8, doi: 10.1155/2012/206857.

[6] J. Nagel, E. Tkacz, and S. Reddy, "Continuous representation of unevenly sampled signals - An application to the analysis of heart rate variability," Biomedical Engineering Applications, Basics \& Communications, vol. 2, pp. 869-877, 1994.

[7] C. L. Nikias and M. R. Raghuveer, "Bispectrum estimation: A digital signalprocessing framework," in Proc. IEEE, July 1987, vol. 75, pp. 869-891.

[8] C. L. Nikias and J. M. Mendel, "Signal processing with higher-orderspectra," IEEE Signal Processing Magazine, July 1993, vol. 10 , no. 3 , pp. 10-37.

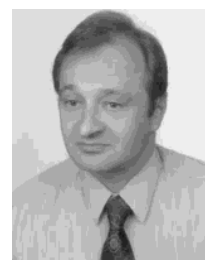

Ewaryst J. Tkacz graduated from Silesian University of Technology, Gliwice, Poland, 1982 with M.Sc. degree in Biomedical Electronics; Ph.D. degree obtained from the Technical University of Brno, Institute of Biomedical Engineering, Czech Republic, 1987 and D.Sc. degree obtained from Institute of Biocybernetics and Biomedical Engineering, Polish Academy of Sciences in Warsaw 1998. Currently he is a professor of Biomedical Engineering at the Faculty of Biomedical Engineering, Department of Biosensors and Biomedical Signals Processing, Silesian University of Technology, Gliwice, Poland and Institute of Biomedical Engineering, Brno 
University of Technology, Brno, Czech Republic. His scientific interests are focused around generally understand biomedical signal processing, neural engineering and combination of both mentioned previously e.g. wavelet neural networks. New methods of HRV signal analysis have been elaborated during Visiting Assistant Professor stay at the Behavioral Medicine Research Center and Department of Biomedical Engineering of the University of Miami, Florida, USA. Recently, also certain aspect of subjects related to control engineering for artificial organs and some aspects of bioinformatics belong to his area of activity.

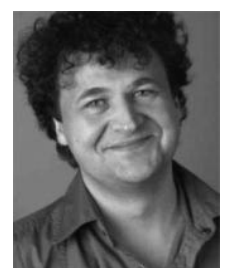

Ivo Provaznik received the $\mathrm{Ph} . \mathrm{D}$. degree in biomedical engineering from the Brno University of Technology, Brno, Czech Republic, in 1991. He was a Visiting Scientist at Johns Hopkins University, B Baltimore, MD. He is currently a professor of biomedical engineering with the Brno University of Technology and the Head of the Department of Biomedical Engineering. He is the author or coauthor of more than 70 scientific papers published in reviewed journals and presented at international conferences indexed in WoS. He supervises bachelor and master's study programs in bioinformatics. His research interests include genomics, bioinformatics, and biomedical signal processing.

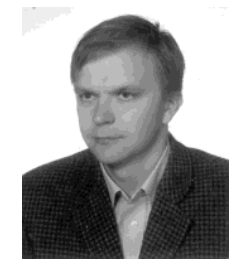

Pawel S. Kostka received the M.Sc. degree in biomedical engineering from Silesian University of Technology, Gliwice, Poland in 1996 and the Ph.D. degree in electronics in 2001 at the same university. Since 1996, he has been a Lecturer with the biomedical Electronics Department in the Silesian University of Technology. As a lecturer, he taught several subjects related to computer programming, statistics, biomedical electronic and numerical methods. His research interest includes biomedical signal processing, artificial intelligence methods application and modeling of biomedical systems. Special interests are focused around cardiac and circulatory system signals, time-frequency analysis and hybrid methods. 